\title{
Lesões do Aquiles - Parte 1: Tendinopatias
}

\section{Achilles Tendon Lesions - Part 1: Tendinopathies}

\author{
Nacime Salomão Barbachan Mansur ${ }^{10}$ Lucas Furtado Fonseca1이 Fábio Teruo Matsunaga10 \\ Daniel Soares Baumfeld ${ }^{2}{ }^{-0}$ Caio Augusto de Souza Nery ${ }^{10}$ Marcel Jun Sugawara Tamaoki ${ }^{10}$
}

${ }^{1}$ Departamento de Ortopedia e Traumatologia, Escola Paulista de
Medicina, Universidade Federal de São Paulo, São Paulo, SP, Brasil
2Departamento de Ortopedia e Traumatologia, Universidade Federal
de Minas Gerais, Belo Horizonte, MG, Brasil

Rev Bras Ortop 2020;55(6):657-664.

\begin{abstract}
Endereço para correspondência Nacime Salomão Barbachan Mansur, $\mathrm{PhD}$, Departamento de Ortopedia e Traumatologia, Escola Paulista de Medicina, Universidade Federal de São Paulo, Rua Napoleão de Barros, $715,1^{\circ}$ andar, Vila Clementino, São Paulo, SP, Brasil (e-mail: nacime@uol.com.br).
\end{abstract}

\section{Resumo \\ Palavras-chave \\ - tendinopatia \\ - tendão do calcâneo/ cirurgia \\ - insercional \\ - não insercional}

As afeç̧ões que acometem o tendão calcâneo são extremamente comuns na população geral e no cotidiano da atenção ortopédica. Sua crescente incidência, motivada pelo envelhecimento da população, pela melhora no acesso à saúde, pelo aumento na utilização de drogas de uso contínuo, pela errática participação esportiva e outros fatores, tem causado impacto direto na sociedade. As tendinopatias, ainda que hoje respaldadas por tratamentos consolidados, carecem de suporte científico de qualidade para muitas das suas recomendações. Novas terapêuticas têm surgido com o objetivo de potencializar o resultado da abordagem não-operatória e diminuir a quantidade de pacientes que necessitam de uma cirurgia. Esses procedimentos operatórios apresentam uma boa resposta álgica e funcional dos pacientes, no entanto não são livres de complicações e dos altos custos que os cercam.

Calcaneal tendon injuries are extremely common in the general population and in orthopedics routine care. Its increasing incidence, which is motivated by an aging population, improved access to the health care system, increased prescription of continuous-use medication, erratic participation in sports and other factors, has had a direct impact on society. Consolidated treatment options for tendinopathies lack quality scientific support for many modalities. New therapies have emerged to enhance nonsurgical approach outcomes and to reduce the number of patients requiring surgery. Although these operative procedures provide good pain relief and functional outcomes, they are costly and may lead to complications.

\section{Introdução}

\section{Tendinopatias do Aquiles}

$O$ tendão calcâneo é o tendão mais forte e espesso do corpo humano. ${ }^{1,2}$ Origina-se da confluência dos músculos sóleo e gastrocnêmio (possuidor de dois ventres) e toda a unidade é denominada tríceps sural. ${ }^{3}$ A cerca de seis centímetros (proximais) de sua inserção na tuberosidade posterior do osso calcâneo, esses ventres formam o tendão. Nesse trajeto, ele apresenta uma rotação de cerca de 180 graus de sua

recebido

10 de Setembro de 2019 aceito

29 de Novembro de 2019
DOI https://doi.org/

10.1055/s-0040-1702953. ISSN 0102-3616. estrutura, determinando que as fibras provenientes do sóleo estejam mediais e as do gastrocnêmio laterais na sua porção distal. Essa rotação confere maior força e resistência ao tendão ao diminuir a friç̧ão entre as fibras e suas distorções. No entanto, pode comprometer a vascularização local. ${ }^{4}$

A topografia de dois a seis centímetros proximais da inserção é designada região não-insercional. Apresenta baixa vascularização por conta da longitude em relação às porções miotendínea e óssea, conferidoras de aporte nutricional adicional. Essas particularidades anatômicas a predispõem
Copyright $\odot 2020$ by Sociedade Brasileira License terms de Ortopedia e Traumatologia. Published by Thieme Revinter Publicações Ltda, Rio de Janeiro, Brazil 
à ocorrência de doenças degenerativas e rupturas. Distalmente, o tendão tem uma anatomia particular, também favorecedora do desenvolvimento de tendinopatias. A alcunha de região insercional pressupõe os dois centímetros distais do tendão até a sua implantação no os calci. Nessa localização, o Aquiles é balizado por uma bursa anterior (retrocalcaneana) e uma bursa posterior (pré-aquileana ou subcutânea) no plano sagital. Sua inserção na tuberosidade é especializada, espraiada, tipo leque, com fibras que se estendem tanto no plano lateral, quanto medial e distal. ${ }^{1}$

Essa êntese é extremamente singular e considerada por alguns autores como um órgão particular. É formada pela junção osteotendínea, coberta por uma fibrocartilagem sesamoide adjacente ao tendão, pelo periósteo dorsal da tuberosidade e pelo ápice da gordura de Kager. As bursas estão distais nessa topografia. ${ }^{2,5}$ A microanatomia do tendão calcâneo respeita a organização de outros tendões do corpo humano. O componente celular é formado por tenócitos e tenoblastos em até 95\% dos seus elementos. Essas células têm diferentes tamanhos e formas, sendo dispostas em cadeias longas e paralelas. O elemento extracelular é composto em $90 \%$ por tecido colágeno, com predominância do tipo I (95\%), organizado em bandas paralelas mantidas por pequenas moléculas proteoglicanas. Cerca de $2 \%$ do elemento é composto de elastina, que confere até $200 \%$ de capacidade de deformação antes da falha. O envelhecimento e a incapacidade corpórea em prover cicatrização ideal a esses tecidos modificam essa configuração, promovendo o acúmulo de mucina, fibrina, e colágeno dos tipos III e VII. ${ }^{2,6}$

A tendinopatia do calcâneo é classificada de acordo com seu sítio anatômico em insercional e não-insercional. A tendinopatia insercional do Aquiles (TIA) ocorre na implantação do tendão na tuberosidade do osso calcâneo a até dois centímetros proximais a essa. Está geralmente associada ao entesófito de tração (esporão superior), à deformidade de Haglund (pump bump) e às bursopatias pré e retroaquileana. A diferenciação entre os dois sítios da doença não é meramente topográfica, sendo consensual na literatura. Existem diferenças na epidemiologia das duas condições, assim como teorias etiológicas díspares com algumas similaridades. Apesar de poderem coexistir tanto de modo assintomático quanto sintomático (tendinopatia mista do calcâneo), são consideradas enfermidades distintas na apresentação clínica e no tratamento. ${ }^{7,8}$

\section{Fisiopatogenia}

Degenerações intratendíneas secundárias a quadros inflamatórios de baixo grau e pobre cicatrização biológica caracterizam a tendinopatia do calcâneo. Múltiplas hipóteses foram levantadas para a etiologia dessa doença, dentre elas a sobrecarga (overuse), o desequilíbrio muscular, o mau alinhamento, e o decréscimo no aporte sanguíneo e na forca tênsil com o decorrer do envelhecimento. Atualmente, acredita-se que a origem seja multifatorial, com fatores mecânicos, vasculares, neurais e genéticos desempenhando diferentes papéis nesse processo. A ocorrência de doenças sistêmicas e a utilização de certas drogas também exercem influência adjuvante no desenvolvimento dessa condição. $^{9}$
0 fator extrínseco mais tradicionalmente associado às tendinopatias, em particular a do Aquiles, é a sobrecarga. Microlesões, induzidas pela atividade, que ultrapassam a capacidade regenerativa do tecido, explicam a incidência bimodal dessas doenças. Pacientes jovens praticantes de atividades físicas de alta demanda e pessoas de meia idade que já apresentam um potencial de cicatrização comprometido são as populações mais acometidas por essa afecção. ${ }^{4,9-11}$

Pé cavo, pé plano, instabilidade do tornozelo, sexo e sobrepeso também são relacionados ao desenvolvimento da degeneração intratendínea. Alguns autores citam esses fatores como risco para a doença, em que pese a sobrecarga mecânica inerente a essas condições. Na forma não-insercional da doença, a presença do tendão plantar delgado em íntimo contato com o tendão e com uma cinemática diferente do Aquiles, foi apontada como um indutor da doença. A herança genética, além da clara relação com algumas doenças do metabolismo do colágeno, tem papel relevante na tendinopatia à medida que se identifica a presença da degeneração do Aquiles em familiares, assim como a ocorrência de polimorfismos combinados e genótipos nas populações estudas. ${ }^{12,13}$

Comorbidades, como artrite reumatoide, artrite reativa, espondiloartropatias, lúpus, doenças de depósito (gota e pseudogota), diabetes mellitus, amiloidose, hipercolesterolemia, nefropatias, síndrome de Marfan, doença de EhlersDanlos, hemocromatose e osteogênese imperfecta foram secundariamente relacionadas às doenças dos tendões nas últimas décadas. Drogas das classes das quinolonas, estatinas, corticoides, esteroides anabolizantes e anti-inflamatórios não hormonais também têm sido apontadas em estudos como promotoras de tendinopatias em decorrência da sua inibição da síntese natural de colágeno. Entretanto, uma revisão sistemática recente apontou apenas o abuso de álcool e o uso de ciprofloxacinas como fatores de risco sistêmico com evidência forte para o desenvolvimento de tendinopatias do Aquiles. ${ }^{14,15}$

\section{Tendinopatia Não-Insercional do Aquiles (TNIA)}

\section{Epidemiologia}

A forma não-insercional da doença tem uma incidência de cerca de 1,85 para cada 1.000 habitantes e é responsável por 6 a $17 \%$ das lesões de corredores. ${ }^{4,16}$ A prevalência dessa afecção é estimada em $0,2 \%$ de pacientes sedentários e $9 \%$ de atletas, apresentando característica crescente nas últimas décadas. Os custos do tratamento giram em torno dos $€ 2.500,00$ por paciente no tratamento conservador, valores esses que podem ser multiplicados por seis quando a resolução operatória é considerada. ${ }^{17,18}$

\section{Clínica}

Os pacientes comumente procuram atendimento com queixa de dor na região do tendão ( 2 a $6 \mathrm{~cm}$ proximais da inserção) após as atividades e que, com o tempo, podem acontecer durante a prática esportiva ou laboral. Não é incomum a ocorrência de rigidez na região, o que até pode anteceder o aparecimento da dor. $\mathrm{O}$ aumento de volume é principalmente notado nos indivíduos com condições mais crônicas. ${ }^{4,19}$

O exame físico deve contemplar a avaliação do alinhamento do paciente na procura de deformidades (pé cavo, pé 
plano, doenças congênitas). O diagnóstico é clínico e estabelecido pela existência de dor à palpação do corpo do tendão, que pode também se demonstrar hipertrofiado. A ocorrência de dor medial no tendão é altamente sugestiva da presença do tendão plantar delgado. Alguns pacientes também podem exibir dor na palpação da gordura de Kager. Testes como o do arco doloroso (edema que se movimenta na mobilização do tornozelo) e o Royal London Hospital (edema mais doloroso na extensão) foram descritos, entretanto não são necessários para o diagnóstico e não modificam o planejamento. ${ }^{19}$

\section{Propedêutica Armada}

Os exames subsidiários não são necessários para o diagnóstico, contudo podem ser lançados para o planejamento e prognóstico de tratamento dos pacientes. Radiografias simples podem mostrar calcificações intratendíneas, sugestivas de doença de longa data. Alterações de sinais intrasubstanciais na ultrassonografia confirmam a doença. A elastografia tem se mostrado promissora na possível identificação de populações suscetíveis à doença e desfechos negativos. ${ }^{4,20}$

A ressonância nuclear magnética (RNM) caracteriza de modo completo a tendinopatia e a presença ou não do tendão plantar delgado. A RNM da perna tem sido estudada em pacientes com essa doença. Mudanças no ângulo de bipenação da musculatura do tríceps sural e lipossubstituição ocorrem em pacientes com condições crônicas do Aquiles. Por essas alterações serem de caráter irreversível, quadros avançados geram incapacidade funcional da unidade muscular. Utilizando a classificação de Goutalier, músculos com substituições gordurosas grau 0 e grau 1 podem receber procedimentos de salvamento. Degenerações grau 2, 3 ou 4 traduzem musculaturas com elevado comprometimento biológico e mecânico, sendo qualquer tentativa de reconstrução do seu tendão inócua. ${ }^{21,22}$

\section{Tratamento Não-Cirúrgico}

O tratamento conservador para as tendinopatias não-insercionais do Aquiles apresentava resultados desanimadores até o final do século passado. Os resultados insatisfatórios (29$52 \%$ ) de inúmeras estratégias perduraram por décadas passadas até que Alfredson et al. ${ }^{23}$ estabelecerem um protocolo de fortalecimento excêntrico ( $\mathrm{FE}$ ) do tendão do Aquiles para a forma não-insercional da doença. Esse protocolo consiste em séries de exercícios com 180 repetições por dia, realizados na borda de um degrau, todos os dias da semana por 3 meses (-Figura 1). Os autores obtiveram uma taxa de $82 \%$ de bons resultados. Por conseguinte, corroborados por estudos clínicos e de imagem, conseguiram demonstrar efeito direto na neovascularização do tendão e na sua estrutura macroscópica. Já no início deste século, o protocolo Alfredson de FE era considerado o padrão-ouro no tratamento da TNIA. Ainda hoje, apresenta grau de recomendação A (boa evidência baseada em estudos nível I com resultados consistentes) para o tratamento inicial dessa condição. ${ }^{24,25}$

Alguns artigos confrontaram o protocolo original com modificações (uma vez ao dia, três vezes na semana), com o fortalecimento concêntrico e com o treinamento resistido.

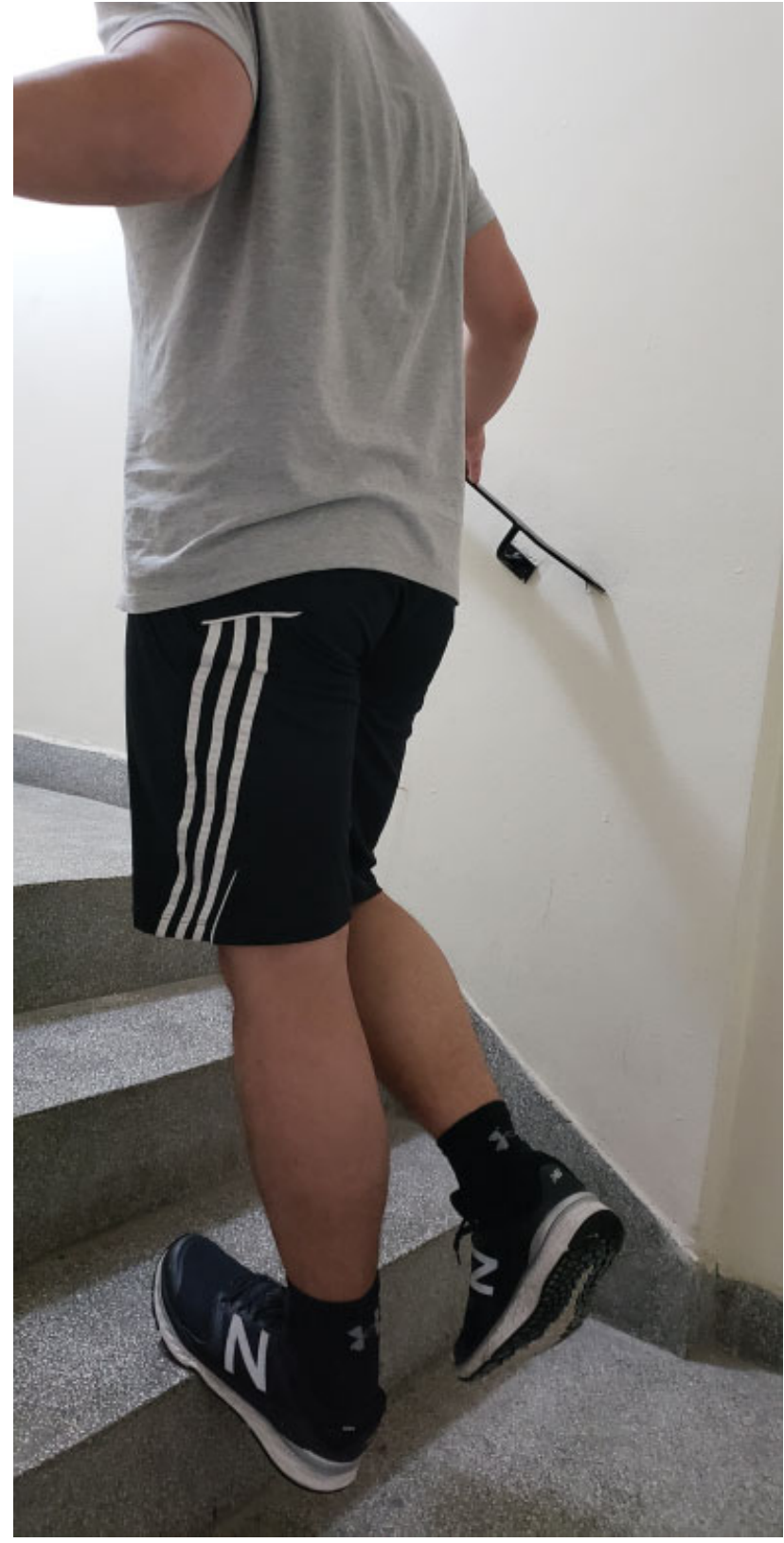

Fig. 1 Exemplo de exercício excêntrico original para tratamento da tendinopatia não-insercional do Aquiles como descrito por Alfredson et al. ${ }^{23}$

Os resultados, ainda que similares, foram amparados por pouquíssimos estudos de qualidade moderada. ${ }^{24,26}$

A correção de fatores etiológicos associados, tais como o gesto esportivo, a sobrecarga, e a utilização de drogas tenotóxicas, deve ser estabelecida quando possível. Infiltrações com plasma rico em plaquetas (PRP), sangue autólogo ou agentes esclerosantes não encontram respaldo na literatura atual de acordo com estudos de moderada qualidade realizados..$^{27,28}$ A utilização de corticoesteroides persiste contra-indicada pelo risco de rupturas do Aquiles. ${ }^{29}$

O tratamento com terapia por ondas de choque (TOC) oferta grau de recomendação B (evidência razoável baseada em trabalhos nível II e III) para tratamento da TNIA. Revisões sistemáticas recentes têm mostrado bons resultados com as 
ondas de choque radiais, apresentando uma alternativa viável na falha do fortalecimento. ${ }^{30,31}$

\section{Tratamento Cirúrgico}

Cerca de 25 a 30\% dos pacientes com TNIA evoluem para o tratamento operatório em decorrência da falha das abordagens conservadoras, o que comumente é decretado após pelo menos 6 meses de boa aboradgem não-operatória. Todas as técnicas aventadas apresentam, no máximo, grau de recomendação $C$ (evidência pobre ou conflituosa baseada em trabalhos nível IVe V) e sustentam resultados positivos (auto relatados) em cerca de $83 \%$ dos casos. Entretanto não são isentas de possíveis complicações, taxas essas de 6,3\% em média, podendo chegar a $42 \%$ em algumas séries de casos. Pacientes com comorbidades graves e doenças que comprometem a vasculatura local apresentam contraindicações claras às operações. ${ }^{18,32}$

A cirurgia busca, como muitas das modalidades não cirúrgicas, a indução de uma reposta cicatricial organizada em consequência da manipulação dos tecidos locais. Além disso, procura remover os neovasos e neonervos que se estabelecem na porção anterior do tendão e contribuem para a gênese da dor e da degeneração. A tenoplastia aberta é o procedimento classicamente utilizado, à medida que permite ao cirurgião ressecar o tecido intratendíneo doente. Taxas de sucesso que variam entre 36 e $100 \%$ (média $78,9 \%$ ) foram reportadas. ${ }^{33}$

A retirada exagerada de tecido degenerado pode enfraquecer o tendão e aumentar sua susceptibilidade a rupturas. A tradicional recomendação de se reforçar, reconstruir ou transferir tendões quando o Aquiles apresenta degeneração superior a $50 \%$ ou é ressecado em volume acima a 50\% é extremamente empírica e não encontra respaldo na literatura. ${ }^{34,35}$ A presença subjetiva de uma grande parcela de tendinopatia deve ser manejada com procedimentos de salvamento do tendão a depender da qualidade da musculatura do tríceps sural. Lipossubstituições grau 0 ou 1 permitem que o Aquiles seja reconstruído com um enxerto livre, como por exemplo o tendão semitendíneo. Músculos em grau 2, 3 ou 4 de Goutalier (ou tendões com aderência extrema do coto proximal) devem ser abandonados, e uma transferência tendínea, como por exemplo a do flexor longo do hálux (aberta ou endoscópica), deve ser efetuada. ${ }^{21,22,36}$

Procedimentos com menor invasividade estão indicados em quadros iniciais e mais frustros da doença. A tenoscopia do Aquiles com desbridamento anterior do tendão foi relatada como portadora de bons resultados, apesar das pequenas séries publicadas. ${ }^{37}$ A tenotomia do plantar delgado, associada ou não ao procedimento tenoscópico (-Figura 2), também tem demonstrado resultados animadores não obstante a ausência de bons estudos que a suportem. ${ }^{38} \mathrm{O}$ alongamento proximal do gastrocnêmio, procura aliviar o encurtamento da cadeia muscular posterior e apresenta até $92 \%$ de acordo com poucos estudos específicos. ${ }^{39}$ Tenotomias percutâneas do Aquiles nas áreas doentes, com objetivo de se estimular cicatrização local, mostraram até $75 \%$ de sucesso segundo algumas séries pequenas de casos. ${ }^{40} \mathrm{O}$ descolamento anterior do tendão de modo minimamente invasivo, utilizando um fio de sutura calibroso, foi relatado como eficaz a curto e longo prazo. ${ }^{41} \mathrm{O}$ resumo das indicações das modalidades de trata-

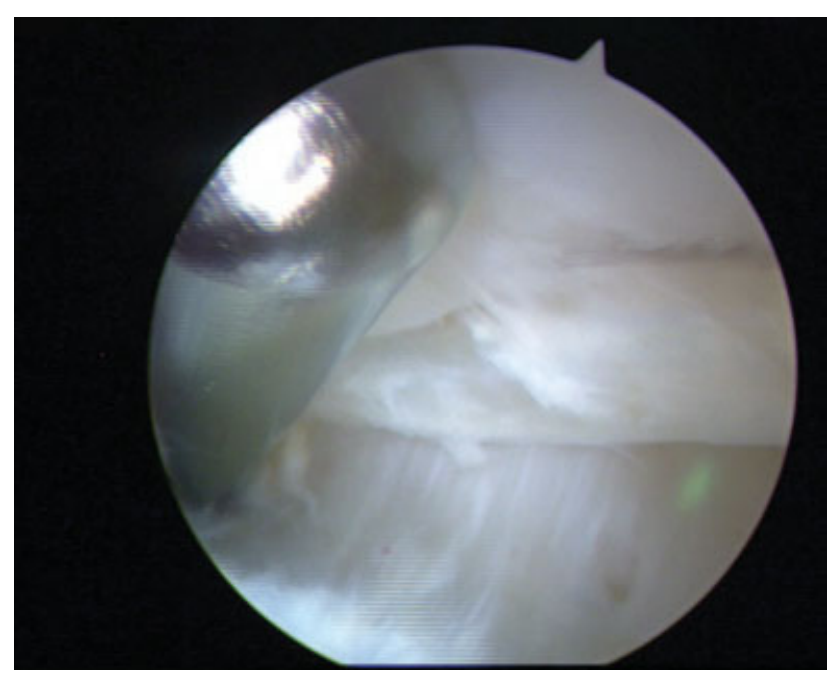

Fig. 2 Tenoscopia do Aquiles para desbridamento da sua região anterior e tenotomia do plantar delgado.

Tabela 1 Grau de recomendação dos tratamentos para a tendinopatia não insercional do Aquiles

\begin{tabular}{|l|l|}
\hline Modalidade & Grau de recomendação \\
\hline Fortalecimento excêntrico & A \\
\hline Terapia por ondas de choque & B \\
\hline Plasma rico em plaquetas & I \\
\hline Tenoplastia aberta & B \\
\hline Tenoplastia endoscópica & I \\
\hline Transferência tendínea & I \\
\hline Procedimentos percutâneos & I \\
\hline Alongamento do gastrocnêmio & I \\
\hline
\end{tabular}

mento baseado nas evidências científicas atuais encontra-se na - Tabela 1 .

\section{Tendinopatia Insercional do Aquiles}

\section{Epidemiologia}

As tendinopatias insercionais apresentam uma incidência populacional de $3,7 \%$ e correspondem a $25 \%$ de todas as doenças do Aquiles. A prevalência em atletas corredores está entre 5 a $18 \%$. Ocorrem tanto em pacientes sedentários, impactando profundamente suas funções e qualidade de vida, como em esportistas profissionais, causando perda de desempenho e rendimento. Estima-se que 50\% dos corredores terão pelo menos um episódio de tendinopatia insercional durante a vida. Os custos relacionados ao tratamento podem girar em torno das $£ 14.000,00$ por paciente, considerando-se apenas o manejo não-cirúrgico. No entanto, esse valor pode setuplicar quando o desfecho operatório é incluído na análise. ${ }^{42-44}$

\section{Clínica}

Com frequência, os pacientes procuram atendimento com queixa de dor, inchaço, perda de força e rigidez local. Referem dificuldades progressivas para o treinamento atlético, 
simples caminhadas e até atividades do cotidiano. Essa hipersensibilidade local é exacerbada pela utilização de calçados com contraforte justo (que aplicam compressão direta na região da doença) ou pelo aumento da demanda física. O diagnóstico é essencialmente clínico; logo, exames subsidiários, como radiografia (RX), ultrassonografia (US) e ressonância nuclear magnética (RNM), são realizados apenas para confirmação da lesão, exclusão de diagnósticos diferenciais, e possível planejamento operatório. ${ }^{45}$

O diagnóstico da TIA é estabelecido por meio da dor à palpação da região de inserção tendínea no osso calcâneo (e até dois centímetros proximais). A presença de edema local, que pode estar associado à flogose baixa, secundária ao processo inflamatório da bursa pré-aquileana, sustenta a hipótese. 0 teste dos dois dedos (two-fingers test) é descrito para a avaliação da ocorrência da bursite retrocalcaneana e da deformidade de Haglund, sendo positivo quando o paciente relata dor após a compressão da região anterior do tendão, justa proximal ao sítio insercional. 0 teste de Silfverskiöld avalia a(s) estrutura(s) responsável(is) pelo encurtamento da cadeia posterior, condição frequentemente adjunta à tendinopatia do Aquiles. É realizado com o paciente em posição prona, ao observar ou não a melhora da extensão do tornozelo (e do encurtamento dos músculos) na flexão passiva do joelho do paciente. Esses dois últimos testes não são necessários para o diagnóstico da TIA, entretanto, floreiam o cenário e podem ser úteis na escolha do tratamento. ${ }^{9,45,46}$

\section{Propedêutica Armada}

A propedêutica armada pode trazer algumas informações para o prognóstico e planejamento terapêutico dos pacientes com TIA. As radiografias são capazes de demonstrar o aumento de volume de partes moles no local, a ocorrência de um entesófito de tração (esporão superior) e a presença da moléstia de Haglund (pump bump), uma neoformação óssea na superfície superior da tuberosidade do osso calcâneo, anterior à bursa retrocalcaneana. Eventualmente, é possível observar calcificações intratendíneas. A US consegue identificar áreas de degeneração, formação de cálcio e tecido ósseo por meio das suas respectivas sombras acústicas. A RNM é a que melhor define a chamada "pêntade da tendinopatia insercional" pelos radiologistas, pois evidencia de modo claro as duas bursopatias, a doença degenerativa do tendão, o Haglund e o entesófito de tração. Todos esses achados costumam persistir após a evolução natural da doença ou mesmo do tratamento bem-sucedido, não sendo critérios para alta ou sucesso na abordagem da TIA. Alguns pacientes assintomáticos podem exibir esses comemorativos num exame de rotina. ${ }^{47,48}$

\section{Tratamento Não-Cirúrgico}

Tradicionalmente, a abordagem terapêutica inicial é baseada no tratamento não-cirúrgico. A busca por uma melhor resposta nos pacientes com tendinopatia insercional levou diversos autores a estabelecerem o mesmo protocolo excêntrico de Alfredson ${ }^{23}$ para esse grupo. Contudo, mesmo com as modificações descritas por Jonsson et al. ${ }^{49}$ (exercícios realizados no nível do solo, sem a fase de extensão do tornozelo negativa no degrau que pode ser dolorosa para pacientes com TIA), os bons

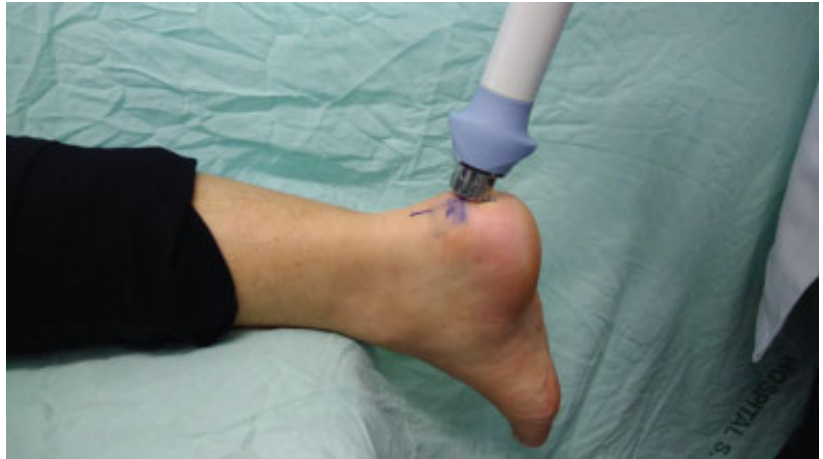

Fig. 3 Posicionamento e aplicação da terapia por ondas de choque radial para tendinopatia insercional do Aquiles.

números da TNIA não foram replicados nessa população. Estudos relataram resultados positivos em apenas 32 a $67 \%$ dos pacientes. Ademais, diversas modalidades de tratamento conservador também se mostraram desapontadoras nas últimas décadas, provendo resultados inclusive inferiores aos do fortalecimento excêntrico (FE). Isso permitiu que, mesmo sob essas circunstâncias negativas, o protocolo Alfredson modificado fosse considerado a referência para a abordagem nãooperatória da TIA, apresentando hoje grau B (evidência razoável baseada em trabalhos nível II e III) de recomendação. ${ }^{9,49-51}$

A terapia por ondas de choque (TOC) utiliza ondas de energia ( Figura 3) que se propagam em três dimensões e induzem um aumento abrupto da pressão intrassubstancial em nanossegundos. ${ }^{52}$ Seu uso busca a indução de respostas intersticiais e extracelulares que levem à regeneração tecidual. Estudos demonstram efeitos diretos dessa terapia na estimulação de fatores cicatriciais. ${ }^{53,54}$ Para as tendinopatias do Aquiles, trabalhos de moderada qualidade apresentaram o procedimento como portador de resultados confiáveis e de riscos muito baixos. Os ensaios clínicos de Costa et al., ${ }^{30}$ Furia, ${ }^{55}$ Rompe et al., ${ }^{56}$ e Rasmussen et al. ${ }^{57}$ Demonstraram bons resultados ( 80 a 88 pontos) e superioridade na comparação com terapias tradicionais (alongamento, manipulação, ultrassonografia), FE, e placebo. A TOC apresenta grau de recomendação B para as TIA. Mais recentemente, novos estudos têm procurado exacerbar a resposta positiva ao tratamento não-cirúrgico pela conjunção de terapêuticas ou introdução de novas alternativas. No entanto, ainda apresentam resultados prematuros e sem validade externa. ${ }^{30,56,58}$

Revisões sistemáticas e artigos de revisão publicados nas últimas décadas escancaram a atual conjuntura em que os tratamentos das tendinopatias insercionais do Aquiles se encontram. As terapêuticas tradicionais não-cirúrgicas (fisioterapia, infiltrações) apresentam resultados inconsistentes e desanimadores. Existe clara recomendação pela prescrição dos exercícios excêntricos no manejo inicial da doença em detrimento da baixa qualidade dos estudos que os suportam. Kearney e Costa $^{46}$ encontraram apenas um artigo ${ }^{55}$ mostrando a efetividade da TOC na TIA, ainda assim com diversas críticas à sua metodologia e avaliações dos desfechos. Al-Abbad e Simon $^{59}$ afirmaram que, apesar da paucidade de trabalhos, o FE deveria ser utilizado como tratamento conservador, e a TOC na sua falha, antes de se indicar uma cirurgia. ${ }^{10,46,60}$ 


\section{Tratamento Cirúrgico}

Os desfechos não muito favoráveis conduziram inúmeros pacientes com TIA à intervenção cirúrgica, com trabalhos relatando essa conduta em 53 a $89 \%$ dos casos. Diferentes técnicas foram descritas para essa condição, desde as mais simples, como a ablação percutânea, o alongamento do gastrocnêmio e o desbridamento artroscópico, até cirurgias mais tradicionais, como a ressecção aberta do tecido degenerado, a reconstrução do tendão com enxerto livre e o uso de transferências tendíneas. $O$ índice de resultados satisfatórios dessas abordagens, apesar de apoiadas em estudos com nível de evidência baixo, encontra-se na faixa dos 75 a 95\%. Todavia, não se demonstraram isentas de complicações, chegando aos $21 \%$ em alguns trabalhos e com uma considerável parcela (cerca de 5\%) evoluindo para novas intervenções e tratamentos continuados. Há de se considerar também o custo elevado de uma cirurgia para TIA, no que concerne os valores relacionados à internação, aos honorários, ao consumo, e aos implantes. ${ }^{61,62}$

Das modalidades de tratamento cirúrgico, apenas a ressecção aberta com reinserção provê grau de recomendação B (baseado em séries de casos), com até até 96\% de bons resultados. ${ }^{35}$ As transferências tendíneas, as ablações percutâneas, o desbridamento endoscópico e as liberações do gastrocnêmio ainda sustentam grau de recomendação I (estudos insuficientes para qualquer recomendação). ${ }^{10}$

A ressecção aberta é corriqueiramente realizada por uma incisão longitudinal ampla, ressecção das bursas, abertura do

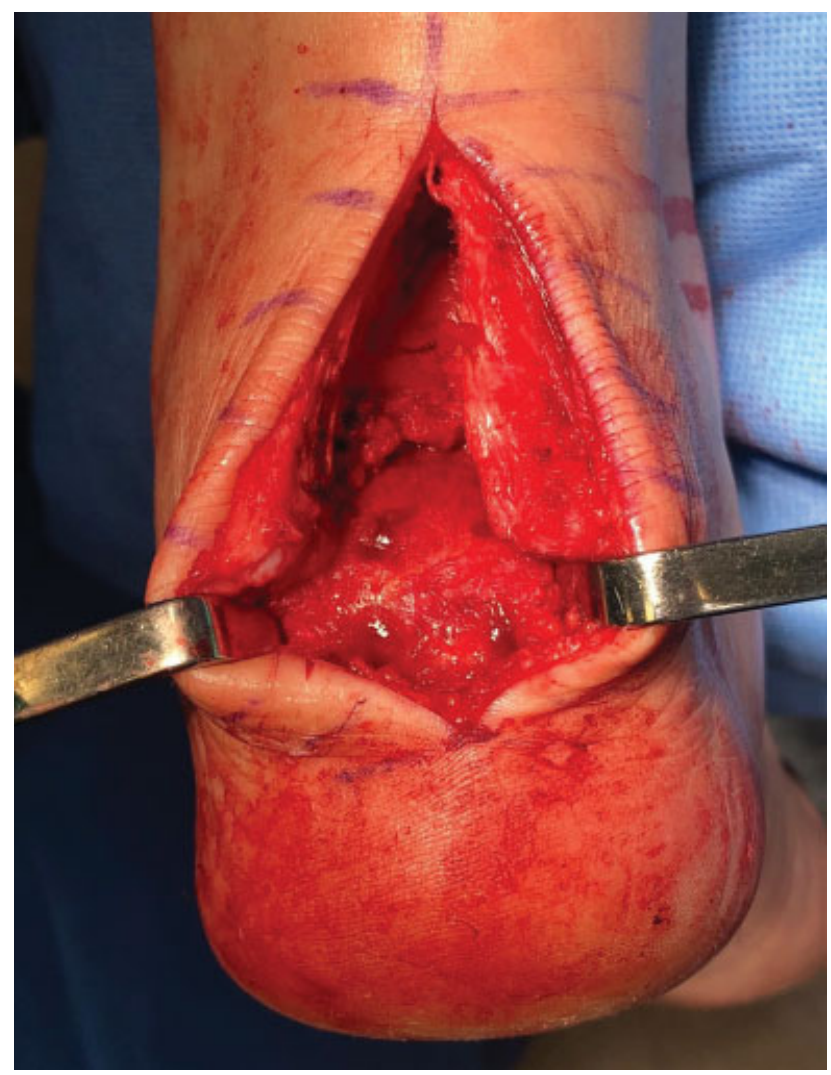

Fig. 4 Exemplo de tenoplastia aberta da inserção do Aquiles com ressecção do entesófito, Haglund e bursas para subsequente reinserção com sistema de ancoras de dupla fileira.
Tabela 2 Grau de recomendação dos tratamentos para a tendinopatia insercional do Aquiles

\begin{tabular}{|l|l|}
\hline Modalidade & $\begin{array}{l}\text { Grau de } \\
\text { recomendação }\end{array}$ \\
\hline Fortalecimento Excêntrico & B \\
\hline Terapia por Ondas de Choque & B \\
\hline Alongamentos & $\mathrm{I}$ \\
\hline Plasma Rico em Plaquetas & $\mathrm{I}$ \\
\hline Agentes Esclerosantes & $\mathrm{I}$ \\
\hline Tenoplastia Aberta com Reinserção & B \\
\hline Tenoplastia Endoscópica & $\mathrm{I}$ \\
\hline Transferência Tendínea & $\mathrm{I}$ \\
\hline $\begin{array}{l}\text { Alongamento Cirúrgico do } \\
\text { Gastrocnêmio }\end{array}$ & $\mathrm{I}$ \\
\hline
\end{tabular}

tendão, retirada do Haglund, remoção dos entesófitos e reinserção com âncoras ( - Figura 4). A análise da quantidade de tendão que pode ser ressecada persiste sendo empírica, apesar da regra dos $50 \%$ ser costumeiramente ignorada por muitos autores. Na presença de tendinopatias avançadas, enxerto livres ou transferências de tendões locais podem ser utilizados de acordo com a qualidade da musculatura do tríceps sural, como discutido no capítulo das tendinopatias não-insercionais. O semitendíneo e o flexor longo do halux são, respectivamente, os doadores de escolha pela proximidade e caracetrísticas biomecânicas. ${ }^{8,21}$

A tentativa de diminuir a morbidade e as complicações decorrentes dos procedimentos tradicionais levou ao desenvolvimento de técnicas de menor invasividade e agressão. 0 debridamento endoscópico ou percutâneo da deformidade de Haglund, da bursa pré-aquileana e da região anterior do tendão surgiu como opção, apesar da sua incapacidade em acessar um possível entesófito ou calcificação intratendínea. $\mathrm{O}$ alongamento do gastrocnêmio proximal também apresenta resultados incipientes no tratamento da TIA. Outras abordagens, como a ablação percutânea e a osteotomia percutânea do calcâneo (Zadek) apenas contam com séries de casos pequenas para suporte. ${ }^{63} \mathrm{O}$ resumo das indicações das modalidades de tratamento baseado nas evidências científicas atuais encontra-se na -Tabela 2.

\section{Considerações Finais}

As tendinopatias do Aquiles são entidades desafiadoras para os pacientes e para toda a cadeia assistencial à medida que leva os agentes envolvidos a longos, custosos e, muitas vezes, ineficazes tratamentos. A forma não-insercional da doença está mais bem amparada por estudos de boa qualidade, que colocam os exercícios excêntricos como excelente modalidade na abordagem inicial desses pacientes, com grau A de recomendação. A falha desse tratamento não-operatório encontra respaldo com grau B para TOCs e novamente grau B para tenoplastia aberta. As infiltrações com substâncias não têm substrato científico de qualidade para seus usos, e as técnicas de menor invasidade ainda se encontram em fase incipiente, apesar dos bons resultados relatados. 
A afecção insercional ainda está cercada de controvérsias, que se estendem desde o tratamento conservador até o cirúrgico. A modificação do protocolo de FE não atingiu os mesmos resultados da TNIA, recebendo grau B de recomendação para a sua prática. Grau esse que se repete para as ondas de choque extracorpóreas e a tenoplastia aberta com reinserção do Aquiles. Modalidades que procuram potencializar os desfechos positivos do tratamento não operatório e cirurgias de menor agressão têm sido propostas a fim de se elevar as taxas de sucesso e diminuir complicações. Porém, ainda apresentam uma paucidade de estudos, e estes são carentes de validação. Esse cenário de incertezas tem recebido atenção da comunidade científica que vem procurando produzir ciência de qualidade que auxilie na seleção do tratamento adequado para essa condição.

Conflito de Interesses

Os autores declaram não haver conflito de interesses.

\section{Referências}

1 Cohen JC. Anatomy and biomechanical aspects of the gastrocsoleus complex. Foot Ankle Clin 2009;14(04):617-626

2 Doral MN, Alam M, Bozkurt M, et al. Functional anatomy of the Achilles tendon. Knee Surg Sports Traumatol Arthrosc 2010;18 (05):638-643

3 Dalmau-Pastor M, Fargues-Polo B Jr, Casanova-Martínez D Jr, Vega J, Golanó P. Anatomy of the triceps surae: a pictorial essay. Foot Ankle Clin 2014;19(04):603-635

4 Longo UG, Ronga M, Maffulli N. Achilles tendinopathy. Sports Med Arthrosc Rev 2009;17(02):112-126

5 Shaw HM, Vázquez OT, McGonagle D, Bydder G, Santer RM, Benjamin M. Development of the human Achilles tendon enthesis organ. J Anat 2008;213(06):718-724

6 Maffulli N, Barrass V, Ewen SWB. Light microscopic histology of achilles tendon ruptures. A comparison with unruptured tendons. Am J Sports Med 2000;28(06):857-863

7 Bernstein DN, Anderson MR, Baumhauer JF, et al. A Comparative Analysis of Clinical Outcomes in Noninsertional Versus Insertional Tendinopathy Using PROMIS. Foot Ankle Spec 2018;12(04): 350-356

8 Roche AJ, Calder JD. Achilles tendinopathy: A review of the current concepts of treatment. Bone Joint J 2013;95-B(10):1299-1307

9 Magnan B, Bondi M, Pierantoni S, Samaila E. The pathogenesis of Achilles tendinopathy: a systematic review. Foot Ankle Surg 2014;20(03):154-159

10 Chimenti RL, Cychosz CC, Hall MM, Phisitkul P. Current Concepts Review Update: Insertional Achilles Tendinopathy. Foot Ankle Int 2017;38(10):1160-1169

11 Vieira CP, Guerra FR, Oliveira LP, Almeida MS, Pimentel ER. Alterações no tendão de Aquiles após inflamação em tecido adjacente. Acta Ortop Bras 2012;20(05):266-269

12 Magra M, Maffulli N. Genetic aspects of tendinopathy. J Sci Med Sport 2008;11(03):243-247

13 Ribbans WJ, Collins M. Pathology of the tendo Achillis: do our genes contribute? Bone Joint J 2013;95-B(03):305-313

14 van der Vlist AC, Breda SJ, Oei EHG, Verhaar JAN, de Vos RJ. Clinical risk factors for Achilles tendinopathy: a systematic review. $\mathrm{Br} \mathrm{J}$ Sports Med 2019:pii: bjsports-2018-099991

15 Marie I, Delafenêtre H, Massy N, Thuillez C, Noblet C; Network of the French Pharmacovigilance Centers. Tendinous disorders attributed to statins: a study on ninety-six spontaneous reports in the period 1990-2005 and review of the literature. Arthritis Rheum 2008;59(03):367-372
16 de Jonge S, van den Berg C, de Vos RJ, et al. Incidence of midportion Achilles tendinopathy in the general population. Br J Sports Med 2011;45(13):1026-1028

17 Yelland MJ, Sweeting KR, Lyftogt JA, Ng SK, Scuffham PA, Evans KA. Prolotherapy injections and eccentric loading exercises for painful Achilles tendinosis: a randomised trial. Br J Sports Med 2011; 45(05):421-428

18 Baltes TPA, Zwiers R, Wiegerinck JI, van Dijk CN. Surgical treatment for midportion Achilles tendinopathy: a systematic review. Knee Surg Sports Traumatol Arthrosc 2017;25(06):1817-1838

19 Singh A, Calafi A, Diefenbach C, Kreulen C, Giza E. Noninsertional Tendinopathy of the Achilles. Foot Ankle Clin 2017;22(04): $745-760$

20 Maffulli N, Via AG, Oliva F. Chronic Achilles Tendon Disorders: Tendinopathy and Chronic Rupture. Clin Sports Med 2015;34 (04):607-624

21 Hoffmann A, Mamisch N, Buck FM, Espinosa N, Pfirrmann CWA, Zanetti M. Oedema and fatty degeneration of the soleus and gastrocnemius muscles on MR images in patients with Achilles tendon abnormalities. Eur Radiol 2011;21(09):1996-2003

22 Fischer MA, Pfirrmann CWA, Espinosa N, Raptis DA, Buck FM. Dixon-based MRI for assessment of muscle-fat content in phantoms, healthy volunteers and patients with achillodynia: comparison to visual assessment of calf muscle quality. Eur Radiol 2014; 24(06):1366-1375

23 Alfredson H, Pietilä T, Jonsson P, Lorentzon R. Heavy-load eccentric calf muscle training for the treatment of chronic Achilles tendinosis. Am J Sports Med 1998;26(03):360-366

24 Sussmilch-Leitch SP, Collins NJ, Bialocerkowski AE, Warden SJ, Crossley KM. Physical therapies for Achilles tendinopathy: systematic review and meta-analysis. J Foot Ankle Res 2012;5(01):15

25 Alfredson $\mathrm{H}$. Clinical commentary of the evolution of the treatment for chronic painful mid-portion Achilles tendinopathy. Braz J Phys Ther 2015;19(05):429-432

26 Silbernagel KG, Thomeé R, Thomeé P, Karlsson J. Eccentric overload training for patients with chronic Achilles tendon pain-a randomised controlled study with reliability testing of the evaluation methods. Scand J Med Sci Sports 2001;11(04):197-206

27 de Jonge S, de Vos RJ, Weir A, et al. One-year follow-up of plateletrich plasma treatment in chronic Achilles tendinopathy: a doubleblind randomized placebo-controlled trial. Am J Sports Med 2011; 39(08):1623-1629

28 Alfredson H, Öhberg L. Sclerosing injections to areas of neovascularisation reduce pain in chronic Achilles tendinopathy: a double-blind randomised controlled trial. Knee Surg Sports Traumatol Arthrosc 2005;13(04):338-344

29 Coombes BK, Bisset L, Vicenzino B. Efficacy and safety of corticosteroid injections and other injections for management of tendinopathy: a systematic review of randomised controlled trials. Lancet 2010;376(9754):1751-1767

30 Costa ML, Shepstone L, Donell ST, Thomas TL. Shock wave therapy for chronic Achilles tendon pain: a randomized placebo-controlled trial. Clin Orthop Relat Res 2005;440(440):199-204

31 Korakakis V, Whiteley R, Tzavara A, Malliaropoulos N. The effectiveness of extracorporeal shockwave therapy in common lower limb conditions: a systematic review including quantification of patient-rated pain reduction. Br J Sports Med 2018;52(06): 387-407

32 Zwiers R, Wiegerinck JI, van Dijk CN. Treatment of midportion Achilles tendinopathy: an evidence-based overview. Knee Surg Sports Traumatol Arthrosc 2016;24(07):2103-2111

33 Tallon C, Coleman BD, Khan KM, Maffulli N. Outcome of surgery for chronic Achilles tendinopathy. A critical review. Am J Sports Med 2001;29(03):315-320

34 Kolodziej P, Glisson RR, Nunley JA. Risk of avulsion of the Achilles tendon after partial excision for treatment of insertional tendonitis and Haglund's deformity: a biomechanical study. Foot Ankle Int 1999;20(07):433-437 
35 Nunley JA, Ruskin G, Horst F. Long-term clinical outcomes following the central incision technique for insertional Achilles tendinopathy. Foot Ankle Int 2011;32(09):850-855

36 Rahm S, Spross C, Gerber F, Farshad M, Buck FM, Espinosa N. Operative treatment of chronic irreparable Achilles tendon ruptures with large flexor hallucis longus tendon transfers. Foot Ankle Int 2013;34(08):1100-1110

37 Pearce CJ, Carmichael J, Calder JD. Achilles tendinoscopy and plantaris tendon release and division in the treatment of noninsertional Achilles tendinopathy. Foot Ankle Surg 2012;18(02): $124-127$

38 van Sterkenburg MN, Kerkhoffs GM, van Dijk CN. Good outcome after stripping the plantaris tendon in patients with chronic midportion Achilles tendinopathy. Knee Surg Sports Traumatol Arthrosc 2011;19(08):1362-1366

39 Duthon VB, Lübbeke A, Duc SR, Stern R, Assal M. Noninsertional Achilles tendinopathy treated with gastrocnemius lengthening. Foot Ankle Int 2011;32(04):375-379

40 Maffulli N, Oliva F, Testa V, Capasso G, Del Buono A. Multiple percutaneous longitudinal tenotomies for chronic Achilles tendinopathy in runners: a long-term study. Am J Sports Med 2013;41 (09):2151-2157

41 Longo UG, Ramamurthy C, Denaro V, Maffulli N. Minimally invasive stripping for chronic Achilles tendinopathy. Disabil Rehabil 2008;30(20-22):1709-1713

42 Amin NH, McCullough KC, Mills GL, Jones MH. The Impact and Functional Outcomes of Achilles Tendon Pathology in National Basketball Association Players. Clin Res Foot Ankle 2016;4(03):205

43 Hutchison A-M, Laing H, Williams P, Bodger O, Topliss C. The effects of a new Tendo-Achilles Pathway (TAP) on an orthopaedic department- A quality improvement study. Musculoskelet Sci Pract 2019;39:67-72

44 Waldecker U, Hofmann G, Drewitz S. Epidemiologic investigation of 1394 feet: coincidence of hindfoot malalignment and Achilles tendon disorders. Foot Ankle Surg 2012;18(02):119-123

45 Den Hartog BD. Insertional Achilles tendinosis: pathogenesis and treatment. Foot Ankle Clin 2009;14(04):639-650

46 Kearney R, Costa ML, Tr F. Insertional achilles tendinopathy management: a systematic review. Foot Ankle Int 2010;31(08): 689-694

47 Stenson JF, Reb CW, Daniel JN, Saini SS, Albana MF. Predicting Failure of Nonoperative Treatment for Insertional Achilles Tendinosis. Foot Ankle Spec 2018;11(03):252-255

48 Krishna Sayana M, Maffulli N. Insertional Achilles tendinopathy. Foot Ankle Clin 2005;10(02):309-320

49 Jonsson P, Alfredson H, Sunding K, Fahlström M, Cook J. New regimen for eccentric calf-muscle training in patients with chro- nic insertional Achilles tendinopathy: results of a pilot study. $\mathrm{Br} \mathrm{J}$ Sports Med 2008;42(09):746-749

50 Fahlström M, Jonsson P, Lorentzon R, Alfredson H. Chronic Achilles tendon pain treated with eccentric calf-muscle training. Knee Surg Sports Traumatol Arthrosc 2003;11(05):327-333

51 Kearney RS, Costa ML. Collagen-matrix allograft augmentation of bilateral rupture of the achilles tendon. Foot Ankle Int 2010;31 (06):556-559

52 Notarnicola A, Moretti B. The biological effects of extracorporeal shock wave therapy (eswt) on tendon tissue. Muscles Ligaments Tendons J 2012;2(01):33-37

53 Wang CJ. Extracorporeal shockwave therapy in musculoskeletal disorders. J Orthop Surg Res 2012;7(01):11

54 Kertzman P, Lenza M, Pedrinelli A, Ejnisman B. Shockwave treatment for musculoskeletal diseases and bone consolidation: qualitative analysis of the literature. Rev Bras Ortop 2015;50(01):3-8

55 Furia JP. [Extracorporeal shockwave therapy in the treatment of chronic insertional Achilles tendinopathy]. Orthopade 2005;34 (06):571-578

56 Rompe JD, Furia J, Maffulli N. Eccentric loading compared with shock wave treatment for chronic insertional achilles tendinopathy. A randomized, controlled trial. J Bone Joint Surg Am 2008; 90(01):52-61

57 Rasmussen S, Christensen M, Mathiesen I, Simonson O. Shockwave therapy for chronic Achilles tendinopathy: a double-blind, randomized clinical trial of efficacy. Acta Orthop 2008;79(02): 249-256

58 Mansur NSB, Baumfeld T, Villalon F, et al. Shockwave Therapy Associated With Eccentric Strengthening for Achilles Insertional Tendinopathy: A Prospective Study. Foot Ankle Spec 2019; ...:1938640019826673

59 Al-Abbad H, Simon JV. The effectiveness of extracorporeal shock wave therapy on chronic achilles tendinopathy: a systematic review. Foot Ankle Int 2013;34(01):33-41

60 Mani-Babu S, Morrissey D, Waugh C, Screen H, Barton C. The effectiveness of extracorporeal shock wave therapy in lower limb tendinopathy: a systematic review. Am J Sports Med 2015;43(03): 752-761

61 Shakked RJ, Raikin SM. Insertional Tendinopathy of the Achilles: Debridement, Primary Repair, and When to Augment. Foot Ankle Clin 2017;22(04):761-780

62 Traina F, Perna F, Ruffilli A, et al. Surgical treatment of insertional Achilles tendinopathy: a systematic review. J Biol Regul Homeost Agents 2016;30(04, Suppl 1):131-138

63 Syed TA, Perera A. A Proposed Staging Classification for Minimally Invasive Management of Haglund's Syndrome with Percutaneous and Endoscopic Surgery. Foot Ankle Clin 2016;21(03):641-664 